J. Clin. Chem. Clin. Biochem.

Vol. 17, 1979, pp. 705-708

\title{
Die kinetische Bestimmung der $\alpha$-Amylase im Serum und Urin mit einem Oligosaccharid als Substrat Modifikation für einen vollmechanisierten Enzymmeßplatz
}

\author{
Von E. Henkel \\ (Eingegangen am 10. Juli/1. Oktober 1979) \\ Dem Gedenken an Professor Dr. Gäbor Szász gewidmet
}

Institut für Klinische Chemie, Abt. II, der Medizinischen Hochschule Hannover, Zentrallabor am Krankenhaus Oststadt

Zusammenfassung: Es wird über eine Modifikation und Adaptation der UV-Methode UltraZyme Plus $\alpha$-Amyl Harleco an den Eppendorf-Enzymautomaten 5010 berichtet.

Aus einem Oligosaccharidgemisch entsteht durch $\alpha$-Amylase Maltose, die durch $\alpha$-Glucosidase zu Glucose gespalten wird. Durch die Hexokinase- und NAD-abhängige Glucose-6-phosphat-dehydrogenase-Reaktion wird Glucose spezifisch erfaßt. Durch Zusatz von Pyruvat, Lactat-dehydrogenase und ATP wird das durch den Abbau hoher Konzentrationen endogener Glucose, z.B. im Harn, entstandene NADH zu NAD oxidiert. Durch Oxamatzusatz wird die Lactat-dehydrogenase-Reaktion gestoppt und danach die $\alpha$-Amylase-Aktivität ermittelt. Bei einem Mittelwert von $65 \mathrm{U} / 1$ beträgt der VK in der Serie $4,02 \%$, die relative Standardabweichung von Tag zu Tag 6,3\%.

Kinetic determination of $\alpha$-amylase in serum and urine with an oligosaccharide as substrate - Modification for a fully mechanized enzyme measuring device

Summary: The author reports a modification of the UV method UltraZyme Plus $\alpha$-Amyl Harleco and the adaptation to the Eppendorf Enzymautomat 5010.

$\alpha$-amylase acts on an oligosaccharide mixture yielding maltose, which is hydrolysed by $\alpha$-glucosidase. The liberated glucose is determined specifically by the hexokinase/glucose-6-phosphate dehydrogenase (NAD ${ }^{+}$-dependent) method. $\mathrm{NADH}$, resulting from the reaction of high concentrations of endogenous glucose, e.g. in urine, is oxidized to $\mathrm{NAD}^{+}$ by addition of pyruvate, lactate dehydrogenase and ATP. Thereafter the lactate dehydrogenase reaction is stopped by addition of oxamate and the $\alpha$-amylase activity is measured.

The CV in the series is $4,02 \%(\bar{x}=65 \mathrm{U} / 1)$, the $\mathrm{CV}$ from day to day $6.3 \%$.

\section{Einfựhrụng}

Zur Bẹstimmung der $\alpha$-Amylase șind eine Reihe von Analysenverfahren gebräuchlich, die sich in vielfacher Hinsicht als unzureichend herausgestellt haben. Die neu entwickelten Bestimmungsmethoden, die Oligosaccharide als Substrat vèrwenden, versprechèn eine Reihe von Vorteilen. Die Molekülgröße des Substrates ist definiert, die Enzymaktivität ist kontinuierlich registrierbar und wird in gebräuchlichen Einheiten ausgedrückt. Der Z̈eitbedarf für die Durchführung eines Tests beträgt wenige Minuten.

\section{Material und Methodik}

\section{Mạterial}

Serum und Urin stammte von stationären und ambulanten Patienten.

\section{Kontrollseren \\ Monitrol I (147B) \\ Monitrol II (58A) \\ Monitrol II (46A) \\ Geräte}

Photometer Eppendorf M 1100 mit Kompensationsschreiber 6511

Eppendorf Enzymautomat 5010

Eppendorf Mikrolitersystem

\section{Reagenzien}

Harleco-UltraZyme Plus $\alpha$-Amyl (Vertrieb AHS-Deutschland Fa. Merz u. Dade, München) (Tab. 1)

Zusatzreagenzien

ATP (Fa. Boehringer Mannheim Best.-Nr. 126888) 0,4 mmol/1 im Testansatz.

Pyruvat (Fa. Boehringer Mannheim Best.-Nr. 127400) 0,4 mmol/l im Testansatz. 
Tab. 1. Testzusammensetzung

\begin{tabular}{|c|c|c|}
\hline & $\begin{array}{l}\text { UltraZyme Plus } \\
\alpha \text {-Amyl }\end{array}$ & Modifikation \\
\hline \multicolumn{3}{|l|}{ Phosphatpuffer pH 6,9 } \\
\hline Oligosaccharidgemisch & $3,5 \mathrm{~g} / 1$ & $3,5 \mathrm{~g} / 1$ \\
\hline Natriumchlorid & $50,0 \mathrm{mmol} / \mathrm{l}$ & $50,0 \mathrm{mmol} / 1$ \\
\hline Magnesiumacetat & $2,5 \mathrm{mmol} / 1$ & $2,5 \mathrm{mmol} / 1$ \\
\hline $\begin{array}{l}\text { Adenosintriphosphat } \\
\text { NAD }^{+}\end{array}$ & $\begin{array}{l}0,83 \mathrm{mmol} / 1 \\
3,0 \mathrm{mmol} / 1\end{array}$ & $\begin{array}{l}1,23 \mathrm{mmol} / 1 \\
3,0 \mathrm{mmol} / 1\end{array}$ \\
\hline Pyruvat & - & $0,4 \mathrm{mmol} / 1$ \\
\hline $\begin{array}{l}\text { Glucose-6-phosphat-dehydro- } \\
\text { genase }\end{array}$ & $2.5 \mathrm{kU} / 1$ & $2.5 \mathrm{kU} / 1$ \\
\hline Hexokinase & $2,5 \mathrm{kU} / 1$ & $2,5 \mathrm{kU} / 1$ \\
\hline$\alpha$-Glucosidase & $300 \mathrm{kU} / 1$ & $300 \mathrm{kU} / 1$ \\
\hline Lactat-dehydrogenase & - & $1,4 \mathrm{kU} / \mathrm{l}$ \\
\hline
\end{tabular}

Lactat-dehydrogenase (Fa. Boehringer Mannheim Best.-Nr. 127230) $1400 \mathrm{U} / 1 \mathrm{im}$ Testansatz.

Oxamidsäure (Fa. Serva Best.-Nr. 31342) $500 \mathrm{mmol} / \mathrm{l}$ im Testansatz.

\section{Reaktionsprinzip}

$\alpha$-Amylase hydrolysiert die Oligosaccharide im Reaktionsansatz zu Maltose, die mit $\alpha$-Glucosidase zu Glucose umgesetzt wird. Glucose wird mit Hexokinase und Glucose-6-phosphat-dehydrogenase (Coenzym: NAD) bestimmt $(1,2)$.

$1 \mathrm{ml}$ Reagenz wird in einer $1 \mathrm{~cm}$ Küvette mit $10 \mu \mathrm{l}$ Serum oder $10 \mu$ Urinverdünnung ( 1 Volumen Urin +2 Volumina Natriumchloridlösung ( $162 \mathrm{mmol} / \mathrm{l})$ gemischt) und die Absorptionszunahme bei $334 \mathrm{~nm}$ nach 10 min Inkubation bei $25^{\circ} \mathrm{C}$ kontinuierlich gemessen.

\section{Auswertung}

$\Delta \mathrm{A} / \min \times 8172=\mathrm{U} / \mathrm{l}$.

\section{Ergebnisse und Diskussion}

Die störende endogene Glucose wird bis zu einer Konzentration von $27,7 \mathrm{mmol} / \mathrm{l}$ in der Inkubationszeit aus dem Reaktionsansatz entfernt. Bis zu einer $\alpha$-Amylaseaktivität von $1000 \mathrm{U} / 1$ verläuft die Reaktion über 20 bis 30 Minuten linear.

Der Verlauf der Kinetik ist an einem Beispiel in Abbildung 1 dargestellt. Auch $\alpha$-Glucosidase spaltet das Substrat; dies entspricht einer $\alpha$-Amylase-Aktivität von $15 \mathrm{U} / 1$, die als Nebenreaktion in Abzug gebracht werden muß. Dieser Wert ist für jede Charge konstant und muß bei Verwendung einer anderen Charge jeweils erneut ermittelt werden. In vier verschiedenen Chargen konnten jedoch bei der Prüfung keine Unterschiede festgestellt werden.

\section{Präzision und Richtigkeit}

Bei Verwendung des modifizierten Testsystems wurde für die Präzision in der Serie $(n=20)$ bei einem Mittelwert von $65,2 \mathrm{U} / 1$ eine relative Standardabweichung von $4,02 \%$ gefunden. Im mittleren Bereich mit einem Mittelwert von $189 \mathrm{U} / \mathrm{l}$ betrug die relative Standardabweichung $3,13 \%$ und im hohen Bereich mit einem Mittelwert von $703 \mathrm{U} / 10,76 \%$.

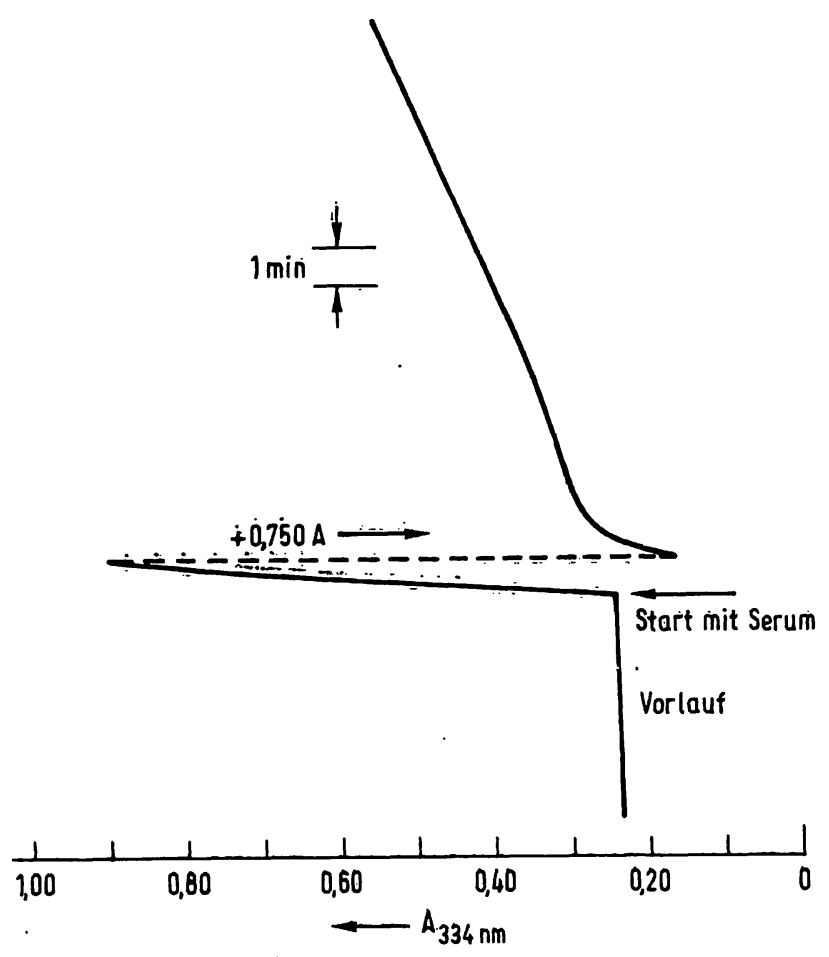

Abb. 1. Schreiber-Diagramm.

Kinetik des UltraZyme $\alpha$-Amyl-Tests. Vorreaktion zur Erfassung des Reagenzien-Leerwertes und Abbau der endogenen Glucose. Darstellung der Phase der Linearisierung der Kinetik durch Degradation des Substrates.

Für die Präzision von Tag zu Ṭa $(n=20)$ im niedrigen, mittleren und hohen Bereich betrugen die relativen Standardabweichungen $6,38 \%, 4,3 \%$ und $2,2 \%$.

Für die Ermittlung der Abweichung vom Sollwert wurden drei verschiedene Chargen Monitrol als Richtigkeitskontrollserum verwendet. Der Mittelwert aus Dreifach-Bestimmungen ergab für Monitrol I 70,2 U/l, die Abweichung vom Sollwert (76,5 U/1) betrug -8,23\%. Monitrol II ergab bei 188,8 U/1 (Sollwert $187 \mathrm{U} / \mathrm{l}$ ) eine Abweichung von $-0,96 \%$. Eine andere Charge Monitrol II ergab für Sollwert und gefundenen Wert übereinstimmende Resultate.

\section{Normalbereich}

Da für die Messung bei $25^{\circ} \mathrm{C}$ Referenzwerte fehlten, wurde an einem Kollektiv zunächst eine orientierende Untersuchung zur Erfassung von Bezugswerten durchgeführt. Sèren von 18- bis 75-jährigen Patienten, die keine Anzeichen für eine Pankreaserkrankung oder Speicheldrüsenerkrankung hatten, wurden untersucht. Dàs Verteilungsdiagramm (Abb. 2) ließ keine geschlechtsspezifischen Unterschiede erkennen. Danach liegt offenbar eine Normalverteilung vor, die einer Bestätigung bedarf. Im gleichen Kollektiv lagen die Enżymaktivitäten mit der Phadebas-Methode im Normalbereich (2). 


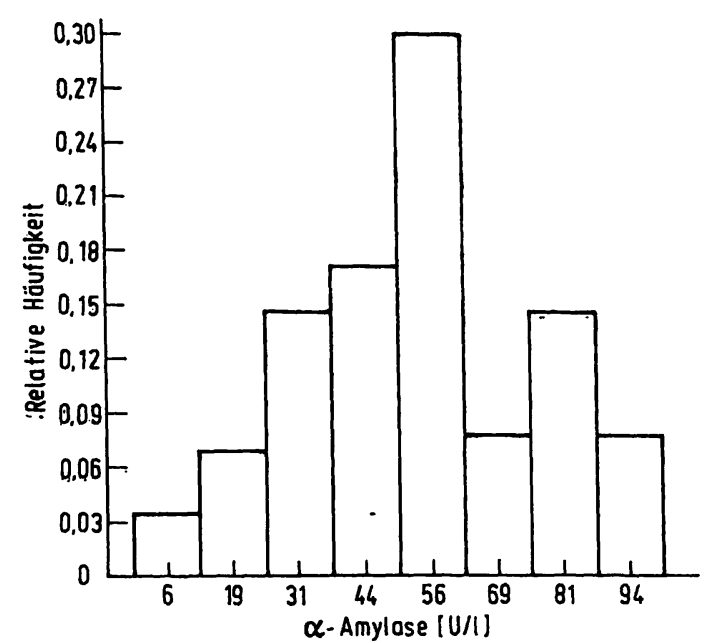

Abb. 2. Histogramm zur Überprüfung des Normalwertes der Ultrazyme Plus $\alpha$ Amyl-Methode.

\section{Stabilitität der Reagenzien}

Das Reagenz ist bei Raumtemperatur 4 Stunden stabil, bei $4{ }^{\circ} \mathrm{C}$ beträgt die Stabilität mindeștenș 20 Ștunden.

Die Oxamatlösung ( $\mathrm{pH} \mathrm{6,8)} \mathrm{iṣt} \mathrm{mindestens} 2$ Monate bei $4{ }^{\circ} \mathrm{C}$ stabil.

\section{Störfaktoren}

Bei Anwendung der Originalvorschrift hattẹ Pyruvat im Serum bis zu $5 \mathrm{mmol} / 1$ keinen Eịnflư Konzentration durch die Lactat-dehydrogenase des Serums und das im Testsystem entstandene NADH innerhalb der Vorinkubationszeit umgesettzt wịd. Hämolyse zeigt erst bei Wẹtẹn ự̣ẹ $10000 \mathrm{mg} / 1$ Hặmọ̣: globin eine Interferenz.

Zusatz von Ascoṛbinsäure ( $5 \mathrm{mmol} / \mathrm{l}$ ) und Methyldopạ $(100 \mathrm{mg} / \mathrm{l})$ stören im Úrin und Serum nicht. Auch die $\alpha$-Glucosidase im Serum und Urin bedịngt kẹnẹn Sṭộr= einfluß.

Die Beimengung gẹinger Mẹngen Phọphhohexoseișo.

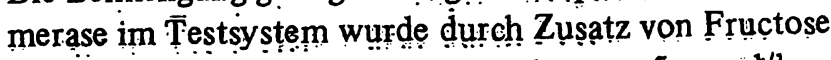
nachgewiesen . Bei einer Konzentration von $5 \mathrm{mmol} / \mathrm{l}$ Fructose im Serum, z.B. nach Infusion von Fructoselösungen, wird die Kinetik der Glụcose-6-phosphaț

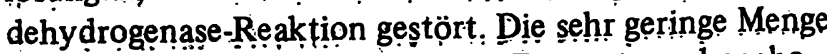
Phosphohexoseisomerase setzt im Testsystem phosphorylierte Fructose um un un eịne geringe Absorptionszunahme ist über die gesamte Reaktionszeit nachweisbar. Bei den getesteten Chargen entsprach die Fremdaktivjtät $16 \mathrm{U} / 1 \alpha$-Amylase-Aktivität. Es ist ạnumerken, daß Fructoselöösungen in dẹ Therapie nụr nọch eine untergeordnete Rolle spielen.

Die Reaktionsgeschwindigkeit der $\alpha$-Amy lase bestimmụngs methoden mit Oligosacchariden hängt sehr von der Kettenlänge dẹr eịngeșetzten Substrate ab. Bei dẹ Ự Ụerprüfung des Substrates von vier Chargen konnten keine Chargenunterschiede festgestellt wẹrdẹn. Die Chargẹn wurden mit Enzympräparationen aus Speichel und Pankreas getestet. Die Ermittlung der Reaktionsgeschwindigkeit mit diesen Enzympräparationen ergab glẹiche Aktivitäten in allen Chargen. Dies erlaubt den Schluß, daß offenbar keine oder nicht nachweisbare Chargenunterschiede bestehen, da nach der Vorinkubation für alle geprüften Chargen gleiche Substratgemische vorliegen.

Mit der Hochdruckflüssigkeitschromatographie wurden im wesentlichen die Substrate Maltopentaose, Maltohexaose und Maltoheptaose im Substratgemisch gefunden. Höhere und niedrigere Oligomere sind nicht in nennenswerten Konzentrationen nachweisbar.

\section{Mechanisierung des Verfahrens}

Die Methode wurde mit einem speziellen Methodenstecker 10-1 an einen Enzymautomaten Eppendorf 5010 adaptiert. Der Stecker 15-1 mit 15 Minuten lnkubationszeit kann ebenfalls verwendet werden, jedoch muß eine Einschränkung des Meßbereichs bei hohen Aktivitäten berücksichtigt werden. Die Dosierervolumina sind $1 \mathrm{ml}$ Reagenz und $10 \mu \mathrm{l}$ Probe. Der Probendurchsatz beträgt 120/Stunde. Urinproben müssșen vor Einsatz im Verhältnis 1:3 verdünnt werden. In Abbildung 3 ist eine Gegenüberstellung der mit der mechanisierten Methode gewonnenen Resultate im Vergleich zur Phadebasmethode dargestellt. Vergleichbare Resultate wurden mit manueller Technik gewonnen.

Beị dẹ Bestimmung der $\alpha$-Amylase im Harn stört endogene Glucose durch hohe Ausgangsabsorption von NADH nach der Vorinkubation. Da dies den Meß-

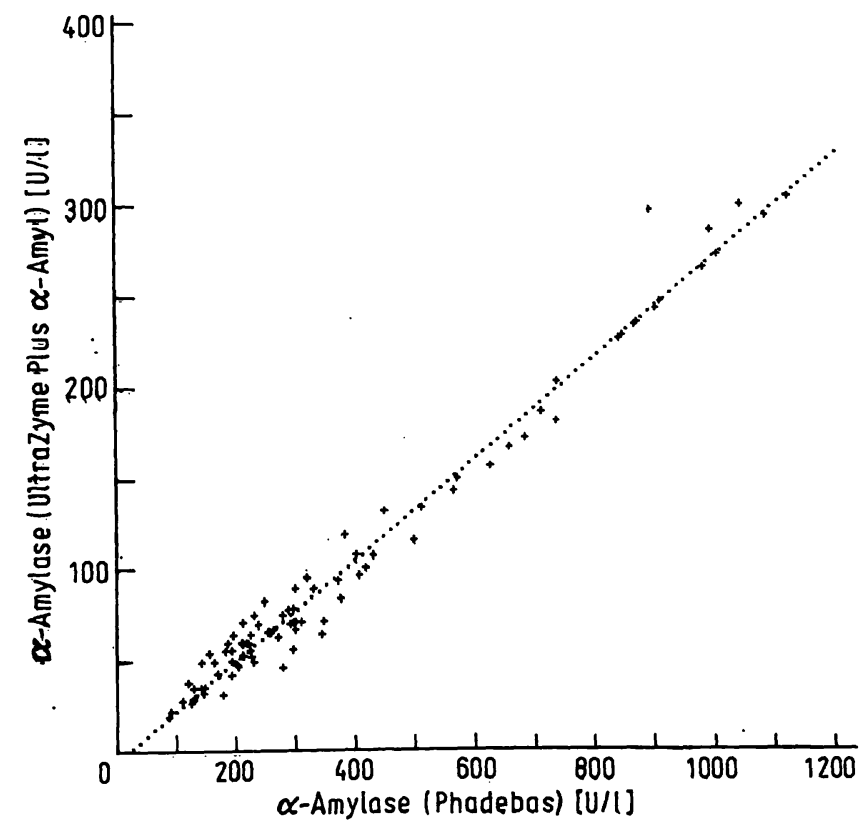

Abb. 3. Vergleich der mit dem Eppendorf-Enzymautomaten $5010 \mathrm{im}$ Ultrazyme-Test enthaltenen Aktivitäten und den mit manueller Technik erzielten Enzy maktivitäten mit der Phadebas-Methode.

$y=-4,16+0,28 x ; r=0,987 ; n=90$ 


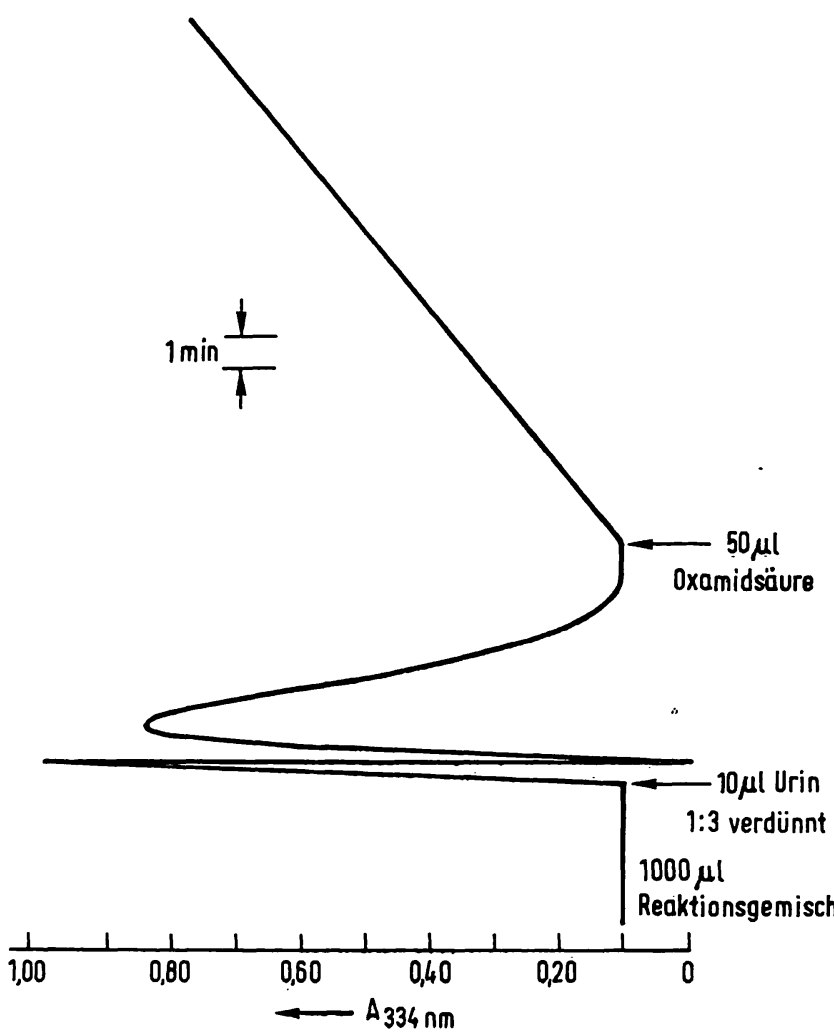

Abb. 4. Schreiber-Diagramm.

Kinetik des Amylasetests Ultrazyme mit Zusatz von Lactat-dehydrogenase, Pyruvat, ATP und Oxamat.

Konzentration an endogener Glucose $160 \mathrm{mmol} / \mathrm{l}$. Amylase 1000 U/1 Harn.

bereich limitiert, wurde folgende Modifikation angewandt:

Dem Ansatz werden 0,4 mmol/1 Pyruvat und $1400 \mathrm{U} / 1$ Lactat-dehydrogenase sowie $0,4 \mathrm{mmol} / \mathrm{l}$ ATP zugesetzt. Mit diesem Zusatz können bis zu $120 \mathrm{mmol} / \mathrm{l}$ Glucose im Harn kompensiert werden. Nach der Vorinkubationszeit wirc die Lactat-dehydrogenase-Reaktion durch $\mathrm{Zu}$ gabe von $50 \mu \mathrm{l}$ einer $500 \mathrm{mmol} / \mathrm{l}$ Natriumoxamatlösung (pH 6,8) gestoppt (Abb. 4).

Bei der Adaptation an den Enzymautomaten Eppendorf wird mit $50 \mu$ l Oxamatlösung durch eine zusätzliche Dosierstation die Lactat-dehydrogenase-Reaktion gestoppt. Die zugesetzten Reagenzien oxidieren NADH zu NAD und das in der Reaktion verbrauchte ATP des Testkits wird durch ATP-Zugabe substituiert. Der Berechnungsfaktor für $334 \mathrm{~nm}$ durch das veränderte Volumen nach der Oxamatzugabe beträgt $\Delta \mathrm{A} / \mathrm{min}$

\section{Literatur}

1. Richter, R., Kemmler, Ardella \& O’Malley, J. A. (1973). Clin. Chem. 19, 644, Abstract.
$X 8657=\mathrm{U} / 1$. Es hat sich gezeigt, daß durch die Modifikation des Reagenz die Ausgangsabsorption eines geringfügig überalterten Reaktionsgemisches auf eine niedrige Absorptionsstufe zurückführt und daß die Reaktion der $\alpha$-Amylase durch die zugesetzten $\mathrm{Re}$ aktionskomponenten in keiner Weise beeinflußt wird. Vergleichsmessungen im Serum mit und ohne Oxamatzusatz sind in Abbildung 5 dargestellt.

Der kommerziell erhältliche Test zur Bestimmung der $\alpha$-Amylase gibt auch im niedrigen Aktivitätsbereich ein genügend großes Signal. Die für den Test benötigte Arbeitszeit liegt in vergleichbarer Größenordnung zu anderen gebräăuchlichen Verfahren zur Enzymaktivitätsbestimmung.

Die Pipettierung von $10 \mu \mathrm{l}$ Probe stellt fựr die Präzision der Methode bei manueller Arbeitsweise ein Problem dar, nicht jedoch für den Einsatz von Automaten und Dilutoren.

Die Reagenzienkosten pro Tëst liegen gegenwärtig bei DM 1,34. Die Modifikation erweist sich beșonders für die Bestimmung der $\alpha$-Amylase im Urin als eine wesentliche Verbesserung in der Praktikabilität der Methode.

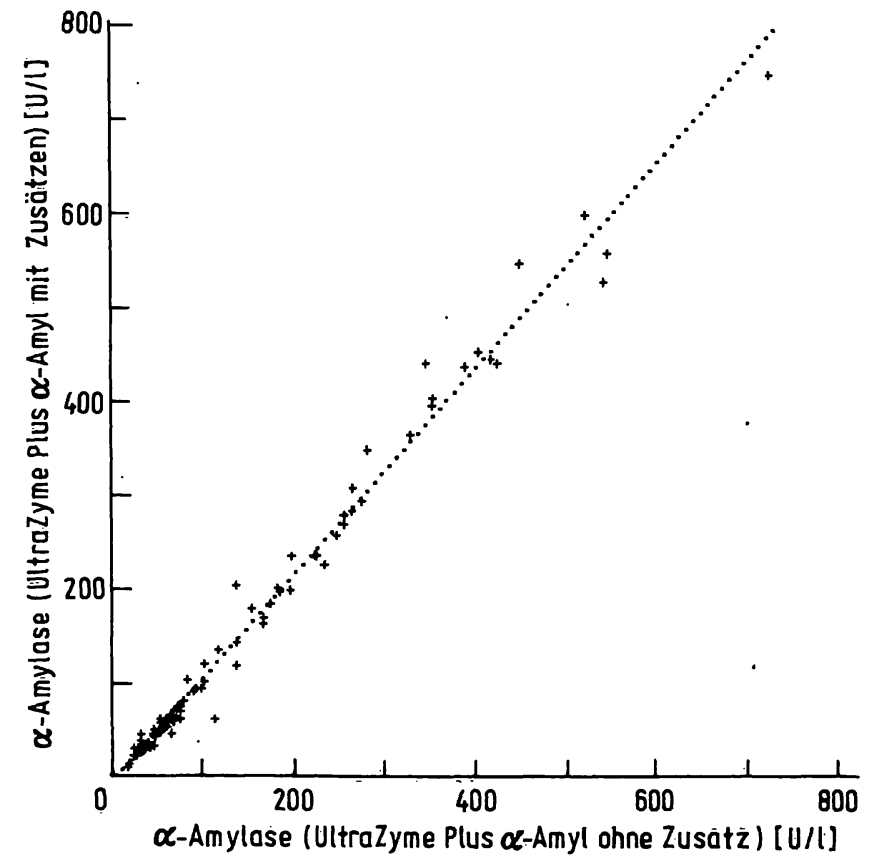

Abb. 5. Methodenvergleich des Ultrazyme $\alpha$ Amyl-Tests mit und ohne Zusatz von Pyruvat, Lactat-dehydrogenase und $\mathrm{ATP}$, sowie Oxamat.

$y=-0,84+1,09 x ; r=0,993 ; n=90$.

2. Mejer, H., Henkel, E. \& Dankert, H. (1979) J. Clin. Chem. Clin. Biochem. 17, 709-716.

Dr. med. E. Henkel

Institut f. Klinische Chemie, Abt. III, Mediz̈inische Hochschule Hannover

Zentrallabor im Krankenhaus Oststadt

Podbielskistr. 380

D-3000 Hannover 51 